\title{
3T MRI Quantification of Hippocampal Volume and Signal in Mesial Temporal Lobe Epilepsy Improves Detection of Hippocampal Sclerosis
}

\author{
A.C. Coan, B. Kubota, F.P.G. Bergo, B.M. Campos, and F. Cendes
}

\begin{abstract}
BACKGROUND AND PURPOSE: In mesial temporal lobe epilepsy, MR imaging quantification of hippocampal volume and T2 signal can improve the sensitivity for detecting hippocampal sclerosis. However, the current contributions of these analyses for the diagnosis of hippocampal sclerosis in 3T MRI are not clear. Our aim was to compare visual analysis, volumetry, and signal quantification of the hippocampus for detecting hippocampal sclerosis in 3T MRI.
\end{abstract}

MATERIALS AND METHODS: Two hundred three patients with mesial temporal lobe epilepsy defined by clinical and electroencephalogram criteria had 3T MRI visually analyzed by imaging epilepsy experts. As a second step, we performed automatic quantification of hippocampal volumes with FreeSurfer and T2 relaxometry with an in-house software. MRI of 79 healthy controls was used for comparison.

RESULTS: Visual analysis classified 125 patients (62\%) as having signs of hippocampal sclerosis and 78 (38\%) as having normal MRI findings. Automatic volumetry detected atrophy in 119 (95\%) patients with visually detected hippocampal sclerosis and in 10 (13\%) with visually normal MR imaging findings. Relaxometry analysis detected hyperintense T2 signal in 103 (82\%) patients with visually detected hippocampal sclerosis and in 15 (19\%) with visually normal MR imaging findings. Considered together, volumetry plus relaxometry detected signs of hippocampal sclerosis in all except 1 (99\%) patient with visually detected hippocampal sclerosis and in 22 (28\%) with visually normal MR imaging findings.

CONCLUSIONS: In 3T MRI visually inspected by experts, quantification of hippocampal volume and signal can increase the detection of hippocampal sclerosis in $28 \%$ of patients with mesial temporal lobe epilepsy.

ABBREVIATIONS: EEG = electroencephalography; HS = hippocampal sclerosis; MTLE = mesial temporal lobe epilepsy

$M^{e}$ esial temporal lobe epilepsy (MTLE) is the most common form of epilepsy in adults. ${ }^{1}$ Although hippocampal sclerosis (HS) is the main pathologic substrate in patients with MTLE, there are several other causes and some patients may have normal MR imaging findings and no specific histopathologic changes de-

Received January 28, 2013; accepted after revision April 10.

From the Neuroimaging Laboratory, Department of Neurology, State University of Campinas, Campinas, São Paulo, Brazil.

Ana C. Coan was responsible for the study concept and design, patient selection and recruitment, MRI visual analysis, interpretation of the data, and manuscript writing. Bruno Kubota contributed the relaxometry analysis; Felipe Bergo, volumetry analysis; Brunno M. Campos, patient recruitment and volumetry analysis; Fernando Cendes, study concept and design, review of patient selection, MRI visual analysis, interpretation of the data, manuscript writing, and reviewing.

This study was funded by São Paulo Research Foundation, grants 2005/56578-4 and 2009/54552-9. Dr Cendes received support from Conselho Nacional de Desenvolvimento Científico e Tecnológico, Brazil.

Please address correspondence to Fernando Cendes, MD, Departamento de Neurologia, Faculdade de Ciências Médicas-UNICAMP, Cidade Universitária Zeferino Vaz, Campinas São Paulo, Brazil, CEP 13083-970; e-mail: fcendes@unicamp.br

O-- Indicates open access to non-subscribers at www.ajnr.org

http://dx.doi.org/10.3174/ajnr.A3640 tected postoperatively. ${ }^{1,2}$ HS can be reliably detected on MRI, ${ }^{2}$ and quantitative analysis of hippocampal formation improves the sensitivity for detecting this pathology. ${ }^{3,4}$ With advances in the MR imaging field in the past decades, the current contributions of these postprocessing MR imaging tools for the detection of hippocampal pathology, especially in tertiary epilepsy centers, are not clear.

The histopathology of HS is characterized by loss of specific neurons and synaptic reorganization of surviving cells, ${ }^{5}$ and in MR images, this is observed as volume reduction and signal hyperintensity in T2 images. ${ }^{6,7}$ Quantification of hippocampal volume has advantages over visual analysis for the detection of subtle and bilateral hippocampal abnormalities. ${ }^{2,8}$ Equally, the quantification of hippocampal T2 signal, especially with relaxometry, can improve the MR imaging diagnosis of HS. ${ }^{9,10}$ Both volume and hippocampal signal quantification measurements have good correlation with histopathologic findings of $\mathrm{HS}^{4}$

Currently, these techniques are considered reliable and reproducible for the detection of hippocampal pathology. ${ }^{11}$ However, 
hippocampal volumetry by manual segmentation is time-consuming and depends on the expertise of the examiner. These 2 features have limited its use in clinical practice. Recently automatic analyses have been shown to be promising; however, there are few studies comparing their efficacy with the visual analyses of high-quality MR imaging by experts in the field. ${ }^{12,13}$

Although it is clear that in studies with $1.5 \mathrm{~T}$ MR imaging, volumetry and relaxometry have significantly higher sensitivity than the qualitative analysis of MR imaging, ${ }^{8,9}$ today most epilepsy centers work with 3T MRI and specific epilepsy protocols, which make the determination of MR imaging signs of HS by visual analysis easier and more accurate. ${ }^{14}$ It has been reported that there is no difference in the hippocampal volume measures of 1.5 and $3 \mathrm{~T}^{15,16}$ and that quantitative measures in 3T MRI can demonstrate ultrastructural details of HS pathology not detectable with lower field scanners. ${ }^{17}$ Once the sensitivity to visually detect signs of HS at $3 \mathrm{~T}$ is higher, ${ }^{14}$ the question that remains is whether the use of hippocampal measurements in these higher field MRIs still adds information to the clinical practice.

In this study, we aimed to evaluate whether the quantification of hippocampal volume and T2 signal in MRI acquired at 3T has a higher sensitivity in detecting MR imaging signs of HS than the visual analysis of these images by epilepsy imaging experts. Our hypothesis was that though the scanners with higher field and specific epilepsy protocols have improved the visual detection of MR imaging signs of HS, the use of techniques to measure the abnormal hippocampal volume and T2 signal can still further improve the detection of HS in patients with otherwise normal MRI findings.

\section{MATERIALS AND METHODS Patients}

This was a prospective study of patients followed at the Epilepsy Clinic of University of Campinas with a clinical and electroencephalographic diagnosis of MTLE according to International League Against Epilepsy criteria, ${ }^{18}$ who consecutively underwent 3T MRI between August 2009 and April 2012. An informed consent form approved by the Ethics Committee of Universidade Estadual de Campinas was signed by all patients before acquisition of MR imaging. Patients with symptomatic MTLE due to lesions other that HS (tumor, vascular malformations, gliosis, focal cortical dysplasia) were excluded.

\section{MR Imaging Epilepsy Protocol and Visual Analysis}

All patients underwent an MR imaging epilepsy protocol with a 3T Intera Achieva scanner (Philips Healthcare, Best, the Netherlands), which included the following:

Coronal images perpendicular to the long axis of the hippocampus, defined at the sagittal image: 1) T2WI multiecho (3mm-thick, no gap, voxel size $=0.89 \times 1 \times 3 \mathrm{~mm}, \mathrm{TR}=3300 \mathrm{~ms}$, $\mathrm{TE}=30 / 60 / 90 / 120 / 150 \mathrm{~ms}$, matrix $=200 \times 180, \mathrm{FOV}=180 \times$ 180, TSE factor $=5$; EPI factor $=5$, flip angle $\left.=90^{\circ}\right) ; 2$ ) T1WI inversion recovery (3-mm-thick, no gap, voxel size $=0.75 \times$ $0.75 \times 3 \mathrm{~mm}, \mathrm{TR}=3550 \mathrm{~ms}, \mathrm{TE}=15 \mathrm{~ms}, \mathrm{TI}=400 \mathrm{~ms}$, matrix $=$ $240 \times 229, \mathrm{FOV}=180 \times 180$, TSE factor $=7$ ), 3) FLAIR (fatsuppressed $=$ spectral-attenuated inversion recovery, fat-suppressed power $=1$, four-mm-thick, section gap $=1 \mathrm{~mm}$, voxel size $=0.89 \times 1.1 \times 2.4 \mathrm{~mm}, \mathrm{TR}=12,000 \mathrm{~ms}, \mathrm{TE}=140 \mathrm{~ms}, \mathrm{TI}=$ 2850 ms, matrix $=180 \times 440, \mathrm{FOV}=200 \times 200)$.

Axial images parallel to the long axis of the hippocampus: FLAIR (fat-suppressed $=$ spectral-attenuated inversion recovery, fat-suppressed power $=1$, four-mm-thick, section gap $=1 \mathrm{~mm}$, voxel size $=0.89 \times 1.1 \times 2.4 \mathrm{~mm}, \mathrm{TR}=12,000 \mathrm{~ms}, \mathrm{TE}=140 \mathrm{~ms}$, $\mathrm{TI}=2850 \mathrm{~ms}$, matrix $=224 \times 160, \mathrm{FOV}=200 \times 200)$.

T1WI volume: isotropic voxels of $1 \mathrm{~mm}$, acquired in the sagittal plane (1-mm-thick, no gap, flip angle $=8^{\circ}, \mathrm{TR}=7.0 \mathrm{~ms}$, $\mathrm{TE}=3.2 \mathrm{~ms}$, matrix $=240 \times 240$, FOV $=240 \times 240)$.

T2WI volume: isotropic voxels of $1.5 \mathrm{~mm}$, acquired in the sagittal plane (no gap, $\mathrm{TR}=1800 \mathrm{~ms}, \mathrm{TE}=340 \mathrm{~ms}$, matrix $=$ $140 \times 140, \mathrm{FOV}=230 \times 230$, TSE factor $=120 ;$ flip angle $=90^{\circ}$; geometry-corrected).

MR imaging sequences were corrected for gradient nonlinearity during the reconstruction step in the scanner.

As a first step, MRI was visually analyzed by 2 epilepsy imaging experts (A.C.C. and F.C.) and the images were classified as having normal findings or signs of HS. Classic signs of HS were as follows: reduction of volume and abnormal shape observed on T1 images and increased signal observed in T2 and FLAIR images. Images were carefully examined by the investigators in light of the clinical and electroencephalography (EEG) data of each patient because this routine of MR imaging evaluation for the investigation of focal epilepsies is used in most epilepsy centers. Special attention was given to rule out subtle signs of focal cortical dysplasia, such as sulcal morphology abnormalities, focal increase of cortical thickness, FLAIR focal signal hyperintensities, or small transmantle signs.

\section{Hippocampal Volumetry}

As the second step, quantification of hippocampal volume and signal was performed. A group of 79 healthy controls (with similar age and sex distribution) was used for comparison (60\% female; median age, 42 years; range, $21-70$ years). Automatic volumetric analysis was performed with FreeSurfer software (Version 5.1.0; http://surfer.nmr.mgh.harvard.edu) by using T1WI volume. Hippocampal volumes were corrected for individual brain (supratentorial) volumes, and corrected hippocampal volumes smaller than 2 SDs (absolute value and/or asymmetry index, defined by the ratio of the smaller over the larger hippocampus of each individual) from the mean of controls were considered as having atrophy.

\section{Hippocampal Signal Quantification}

For signal quantification, we used relaxometry analysis of T2 multiecho images ( 3 -mm sections, $\mathrm{TR}=3300, \mathrm{TE}=30 / 60 / 90 / 120 /$ $150 \mathrm{~ms}$, matrix $=200 \times 176$, FOV $=1802 \times 180)$ with Aftervoxel, a medical image visualization tool written by Felipe Bergo (http://www.liv.ic.unicamp.br/ bergo/aftervoxel). For this analysis, a region of interest was manually defined in 3 different MR imaging sections, including the hippocampus of each individual ( 1 in the head, 1 in the body, and 1 in the tail of the hippocampus) by an investigator blinded to the results of the MR imaging visual evaluation (B.K.). To make the ROI placement more consistent among patients, we defined the section of the hippocampus head as the first in which it was possible to see the temporal horn of the lateral ventricle and therefore to appropriately separate the hippocampal for- 

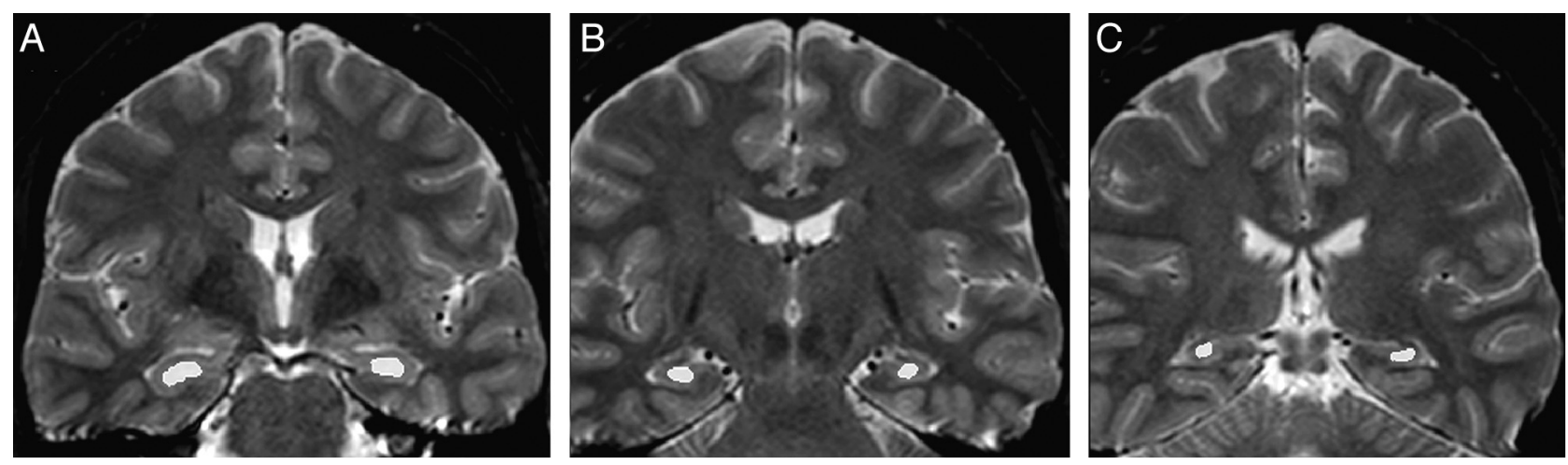

FIG 1. T2 relaxometry in Aftervoxel software. Example of regions of interest manually defined in the head $(A)$, body $(B)$, and tail $(C)$ of the hippocampus of a patient with MTLE.

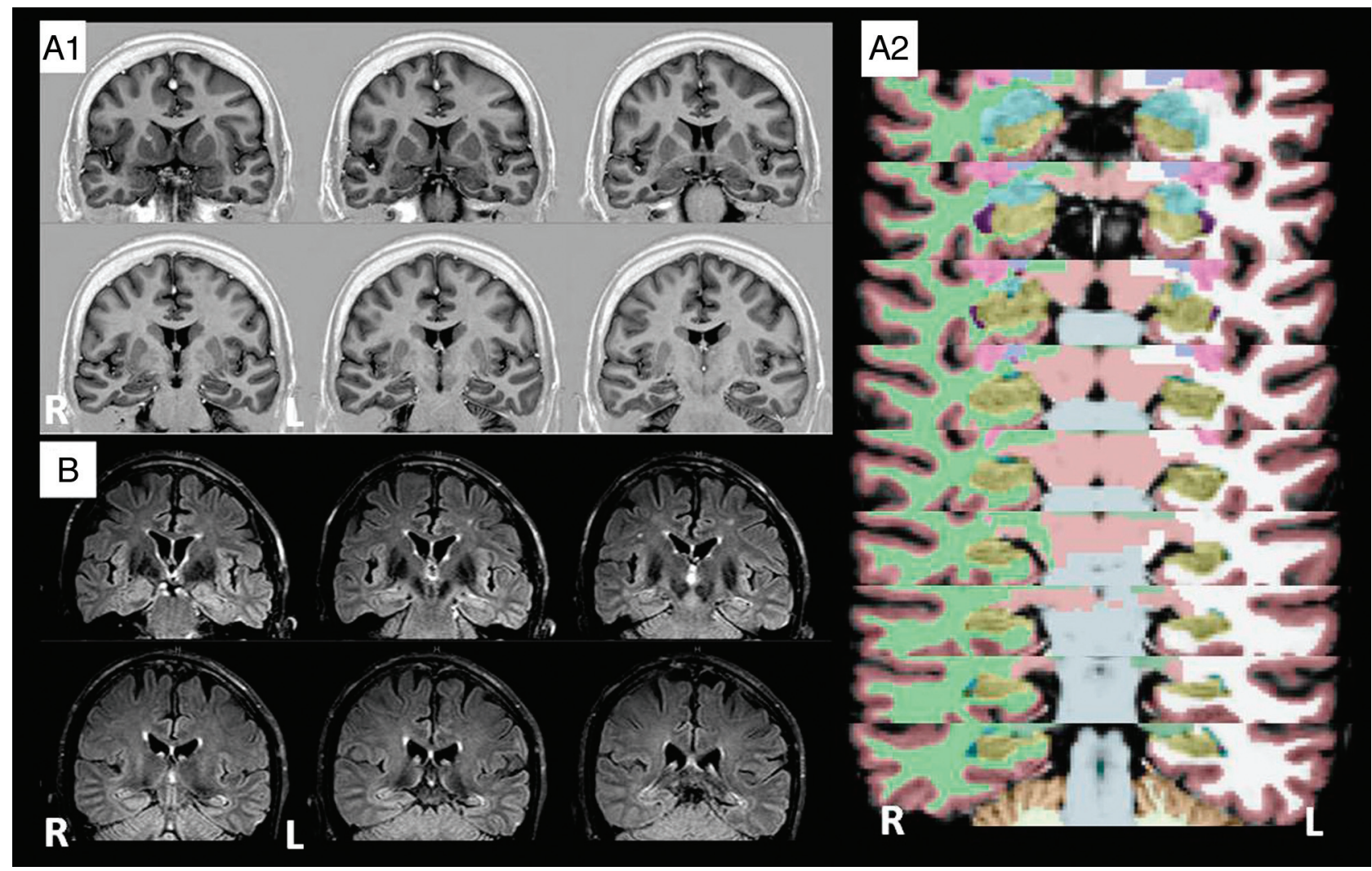

FIG 2. MR imaging signs of HS detected by quantification analysis. Examples of 2 patients with normal MR imaging findings by visual analysis and HS signs detected by quantification techniques. A, A 67-year-old male patient, with MTLE and left EEG seizure focus. His seizures started at 5 years of age and were characterized by an aura described as jamais vu followed by a hipometer complex partial seizure. MR imaging volumetry detected significant left reduced hippocampal volume. A1, TIWI in which both hippocampi were considered normal by visual analysis. A2, FreeSurfer volumetry images in which the hippocampi are shown in yellow. Corrected volume of the left hippocampus: $3.50 \mathrm{~cm}{ }^{3}(z \mathrm{score}=$ -2.20 SDs). B, A 28-year-old male patient with MTLE and left seizure focus. His seizures started at 11 years of age and were characterized by an aura described as fear and an epigastric sensation followed by loss of consciousness and manual automatisms. MR imaging T2 relaxometry detected significant left increased hippocampal signal. Left hippocampus T2 signal: 111.40 ( $z$ score $=3.52$ SDs).

mation from the amygdala. The body of the hippocampus was defined in the fourth coronal section after the region of interest of the hippocampus head, and the tail was defined in the third coronal section after the hippocampus body, in which it was also possible to visualize the quadrigeminal plate. The ROIs were manually drawn to include the larger area of the hippocampus in that section but also to carefully avoid contamination of the hippocampal signal with CSF signal (Fig 1). The mean T2 signal from the 3 sections of each hippocampus was used as the final measurement. Hippocampal signal values higher than 2 SDs from the mean of the control group composed of the same individuals in the volumetry analysis (absolute value and/or asymmetry index, defined by the ratio of higher and lower hippocampal signals of each individual) were considered hyperintense signal.

\section{RESULTS}

Two hundred seventeen patients fulfilled the inclusion criteria. However, after detailed MR imaging visual analysis, 14 patients 
3T MRI signs of HS detected by visual or quantification analysis ${ }^{a}$

\begin{tabular}{lccc}
\hline \multicolumn{1}{c}{ MRI Visual Analysis } & Volumetry & Relaxometry & Volumetry + Relaxometry \\
\hline MTLE-HS (125/203; 62\%) & $119 / 125(95 \%)$ & $103 / 125(82 \%)$ & $124 / 125(99 \%)$ \\
MTLE-NL (78/203; 38\%) & $10 / 78(13 \%)$ & $15 / 78(19 \%)$ & $22 / 78(28 \%)$ \\
Concordance with EEG laterality & $95 \%$ had concordant & $94 \%$ had concordant & \\
& EEG* and 5\% discordant & EEG** and 6\% discordant & \\
\hline
\end{tabular}

Note:-NL, indicates normal MRI.

${ }^{a}$ First column shows the frequency of HS signs detected by visual analysis. The other columns show the percentages of HS signs detected by quantification of hippocampal volume ("Volumetry") and T2 MRI signal ("Relaxometry") alone (second and third columns, respectively) or when considered together (fourth column). In the last line, for each technique the concordance with the laterality of the seizure focus is described as defined by ictal and interictal scalp EEG.

* Excluding the 9 patients and ** the 12 patients with bilateral non-lateralizing EEG.

were thought to have subtle signs of focal cortical dysplasia and were excluded. The final group was then composed of 203 patients (129 female, 74 male; median age, 46 years; range, 17-74 years). There was no difference in sex or age distribution between patients and controls (Sex, $\chi^{2}, P=.527$; age, $t$ test, $P=.072$ ). According to ictal and interictal scalp EEG, 184 patients had unilateral temporal epileptic focus (117 left MTLE and 67 right MTLE) and 19 had bitemporal epileptic focus.

In this group, $176(87 \%)$ patients had drug-resistant seizures, while 27 (13\%) had been free of seizures for at least 2 years.

\section{MR Imaging Visual Analysis}

MR imaging visual analysis detected 125 (62\%) patients with signs of HS (62 left, 54 right, 6 bilateral with left-sided predominance, 3 bilateral with right-sided predominance). For the remaining 78 (38\%) patients, MRI had normal findings by visual analyses. Although the qualitative analysis detected signs of hippocampal sclerosis in $62 \%$ of patients, one cannot say in how many it failed to detect HS because the remaining $38 \%$ of patients may or may not have had HS in their histology, even though they all had MTLE.

\section{Concordance of MR Imaging Visual Analysis and the EEG Epileptic Focus}

From the 125 patients with MTLE with HS signs detected by visual analysis, 117 had unilateral epileptic focus (defined by ictal and interictal EEG); in 88\% (103/117), the side of signs with HS was concordant with the epileptic focus, and it was contralateral in $7.5 \%$ (9/117). In the remaining 4\% (5/117), the MR imaging signs of HS were bilateral and symmetric.

\section{MR Imaging Quantification Analysis}

Automatic volumetry analysis detected hippocampal atrophy in $119(95 \%)$ patients with visual signs of HS and in 10 (13\%) patients with visually normal MR imaging findings. Relaxometry detected hyperintense T2 signal in 103 (82\%) patients with visual signs of HS and in 15 (19\%) patients with visually normal MR imaging findings (Fig 2). Considered together, volumetry plus relaxometry detected signs of HS in all except 1 (124; 99\%) patient with visual signs of HS and in 22 (28\%) patients with visually normal MR imaging findings (Table). Therefore, the use of combined hippocampal volumetry and $\mathrm{T} 2$ relaxometry increased the sensitivity to detect MR imaging signs of HS in $28 \%$ compared with visual analysis.

Volumetry detected bilateral hippocampal atrophy in 9 patients ( 8 with visual signs of HS and 1 with visually normal MR imaging findings). Relaxometry detected bilateral hippocampal hyperintense signal in 22 patients (20 with visual signs of HS and 2 with visually normal MR imaging findings). In patients who had

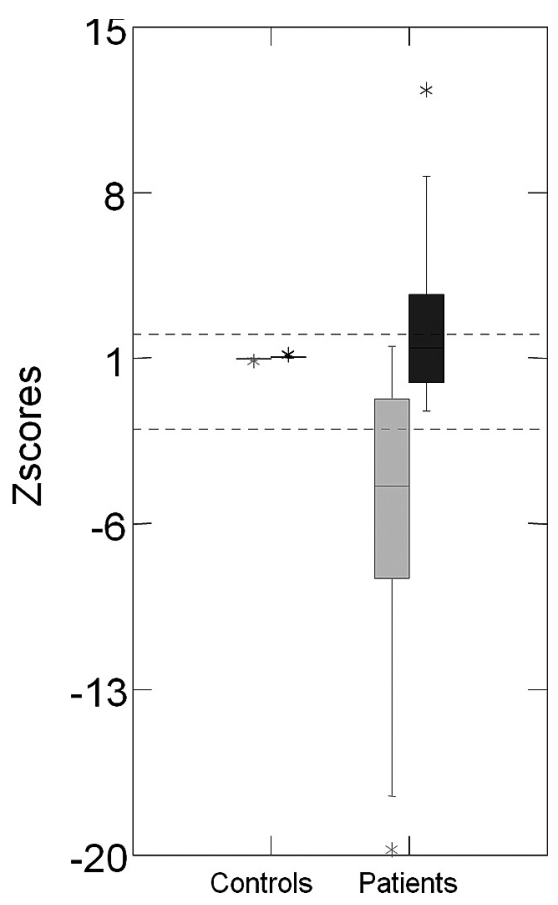

FIG 3. $Z$ score distribution of the hippocampal asymmetry index for volumetry and T2 relaxometry analyses. On the left side is the boxplot graphic of $z$ scores of the volumetry asymmetry index (gray) and T2 relaxometry asymmetry index (black) of controls. On the right side is the boxplot graphic of $z$ scores of the volumetry asymmetry index (gray) and T2 relaxometry asymmetry index (black) of patients with MTLE.

bilateral hippocampal abnormalities detected by the quantifications methods, there was marked asymmetry.

The laterality of the hippocampal abnormality detected by both absolute values and the asymmetry index in volumetry and T2 relaxometry analysis was concordant in all cases. The asymmetry index had a higher sensitivity to detect the hippocampal volume reduction ( $76 \%$ [98 patients] had significant hippocampal volume reduction detected by the absolute volume and 98\% [126 patients] were detected by asymmetry index) and a lower sensitivity to detect T2 hyperintense signal (92\% [109 patients] with significant hippocampal T2 hyperintense signal detected by the absolute volume and $70 \%$ [ 82 patients] detected by asymmetry index). The distribution of the hippocampal asymmetry index for volumetry and $\mathrm{T} 2 \mathrm{re-}$ laxometry in patients and controls is shown in Fig 3.

Figure 4 demonstrates the $z$ score distribution of hippocampal volumes and T2 signal in controls (right and left hippocampus) and patients (hippocampi ipsi- and contralateral to the epileptic focus). 

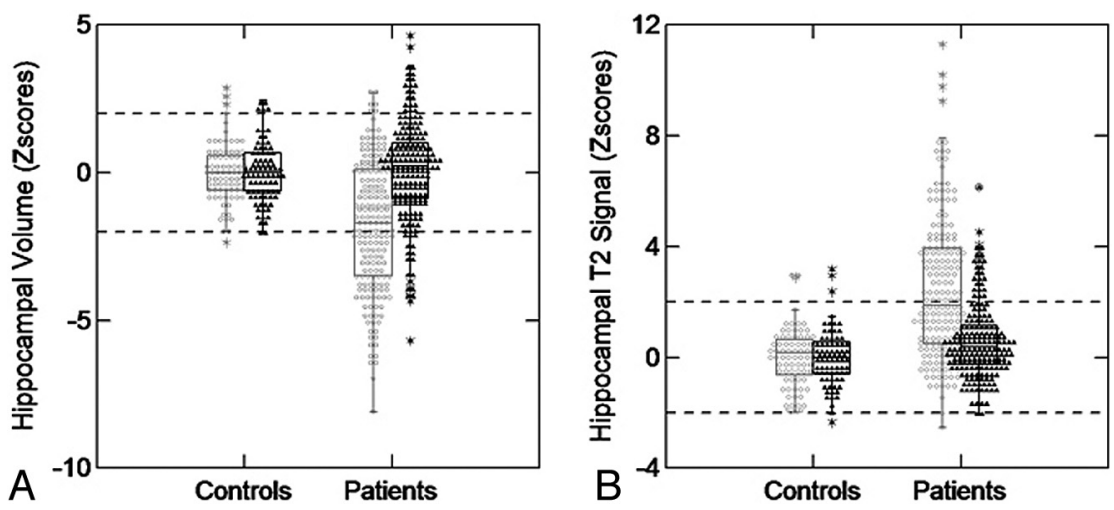

FIG 4. $Z$ score distribution of hippocampal volumes and T2 signal in patients with MTLE and controls. A, Boxplot graphic shows z scores of hippocampal volumetry for controls and patients with MTLE. On the left side of the graphic are shown the hippocampal volumes of controls (white circles = right hippocampi; mean volume, $4.28 \pm 0.35 \mathrm{~cm}^{3}$; black triangles = left hippocampi; mean volume, $4.21 \pm 0.32 \mathrm{~cm}^{3}$ ). On the right side of the graphic are shown the hippocampal volumes of patients (white circles = hippocampi ipsilateral to the epileptic focus; mean volume, $3.78 \pm 0.51 \mathrm{~cm}^{3}$; black triangles $=$ hippocampi contralateral to the epileptic focus; mean volume, $\left.4.28^{3} \pm 0.56 \mathrm{~cm}\right)$. B, Boxplot graphic shows $z$ scores of hippocampal T2 relaxometry for controls and patients with MTLE. On the left side of the graphic is the hippocampal T2 signal of controls (white circles = right hippocampi; mean signal, $96.77 \pm 4.05$; black triangles = left hippocampi; mean signal, $96.98 \pm 4.10$ ). On the right side of the graphic is the hippocampal T2 signal of patients (white circles $=$ hippocampi ipsilateral to the epileptic focus; mean signal, $105.49 \pm 7.88$; black triangles $=$ hippocampi contralateral to the epileptic focus; mean signal, $99.10 \pm 5.01$ ).

\section{Concordance of MR Imaging Visual and Quantification Analysis}

The side of the HS detected by MR imaging visual and volumetry analysis was concordant in all except 1 case (118/119, 99\%). The patient with discordant volumetry and MR imaging visual analysis had a subtle hippocampal atrophy and clear hyperintense T2 signal on the left hippocampus by visual analysis, which was concordant with the $\mathrm{T} 2$ relaxometry.

The side of the abnormal hippocampal signal detected by relaxometry was concordant with the visual analysis in 96\% (99/ 103). In the remaining 4 patients, 2 had bilateral asymmetric hippocampal abnormalities on visual analyses and the relaxometry lateralized to the side with less hippocampal atrophy by both visual and automatic volumetry, and the other 2 patients had unilateral hippocampal atrophy (concordant by visual and automatic volumetry) and the relaxometry lateralized to the side contralateral to the atrophy and hyperintense $\mathrm{T} 2$ signal defined by visual analysis.

\section{Concordance of MR Imaging Quantification Analysis and the EEG Epileptic Focus}

From the 129 patients with MTLE with hippocampal atrophy detected by volumetry, 120 had unilateral epileptic focus (defined by ictal and interictal EEG) and in 95\% (114/120) the side of epileptic focus was correctly lateralized by volumetry. From the 118 patients with MTLE with hippocampal T2 signal hyperintensity detected by relaxometry, 106 had unilateral epileptic focus and in $94 \%$ (100/106) the side of epileptic focus was correctly lateralized by volumetry.

There was no significant difference in the frequency of concordance of signs of HS or hippocampal atrophy detected by visual analysis with the side of the epileptic focus ( $88 \%$ of concordance by visual analyses and $95 \%$ of concordance by volumetry analysis, $\left.\chi^{2}, P=.054\right)$ and no difference in the frequency of concordance of visual signs of HS or hippocampal hyperintense signal detected by relaxometry with the side of the epileptic focus $(88 \%$ of concordance by visual analyses and $94 \%$ of concordance by relaxometry analysis, $\left.\chi^{2}, P=.099\right)$. The small additional gain of the quantification analyses was secondary to better discrimination of the bilateral hippocampal abnormalities.

\section{Concordance of Histopathology and MR Imaging Visual and Quantification Analyses}

Nineteen patients (10\%) underwent surgical treatment (anterior temporal lobectomy or selective amygdalohippocampectomy) due to refractory seizures. Of those, all except 1 individual had confirmed histopathology of HS. In patients with a histopathology of HS, visual analysis and volumetry detected abnormal hippocampi in all cases and relaxometry detected hyperintense signal in $15 / 18(83 \%)$ patients. The patient with a normal hippocampus on histology had no signs of HS detected by either visual or quantitative methods.

\section{DISCUSSION}

The detection of MR imaging signs of HS can help to define seizure etiology and to indicate surgical treatment for patients with drug-resistant MTLE. We demonstrated here that even in 3T MRI analyzed in tertiary centers by epilepsy experts, hippocampal volume and signal quantification can significantly improve the detection of signs of HS in patients with otherwise normal MRI findings by using an epilepsy protocol.

MR imaging has significantly improved the detection of pathologies related to epilepsy. ${ }^{19}$ It is safe, noninvasive, and widely available in epilepsy centers. However, a variable but significant number of patients with focal epilepsies have normal MRI findings and unknown seizure etiology. ${ }^{20-24}$

From the beginning of MR imaging use in epilepsy, special attention has been given to HS because it is the main pathologic feature associated with the most common epilepsy in adults. ${ }^{1}$ Hippocampal volume and signal have been used for research purposes but also in epilepsy clinics to help in the evaluation of drugresistant focal epilepsies. Quantification of hippocampal volume and signal in MRI cannot only detect signs of HS but can consistently help to lateralize the seizure focus in patients with MTLE who are surgical candidates. ${ }^{8}$ For these individuals, the prognosis of surgical resection of the temporal lobe ipsilateral to HS detected by MRI is excellent. ${ }^{21}$ Today, most of the tertiary epilepsy centers have 3T MRI available and visual signs of HS have been more easily detected. ${ }^{14}$ In this context, the contribution of MR imaging quantification methods to detect HS in 3T MRI has not been previously evaluated. Here we demonstrated that with 3T MRI, adequate epilepsy protocols, and expert visual evaluation, quan- 
tification analysis can still improve the detection of subtle signs of HS in $28 \%$ of patients.

The detection of more subtle MR imaging abnormalities in patients with focal epilepsies depends on both the quality of MR imaging acquisition protocol and the experience of the examiner in reading MRIs of patients with epilepsy. A previous study ${ }^{20}$ showed that "nonexperts" reported 61\% of standard MR imaging as having normal findings or as showing no focal abnormality, while epilepsy "expert" examiner reassessments of the same standard MRIs classified $28 \%$ of these scans as technically inadequate and considered only $22 \%$ of these standard MR imaging scans as having normal findings. More important, by using a dedicated epilepsy MR imaging protocol, the same group of "experts" described focal MR imaging abnormalities in $91 \%$ of the same group of patients (they did not include hippocampal volumetry or T2 relaxometry). ${ }^{20} \mathrm{Also}$, in this context, MRIs are always evaluated in the light of clinical, neuropsychological, and EEG data. In this article, we reproduced this optimal visual evaluation of MRI in patients with MTLE: Two epileptologists with expertise in MR imaging evaluation of patients with focal epilepsies reviewed the images of all patients (acquired with an extensive epilepsy protocol) in the context of clinical and EEG data. Even in this most favorable circumstance, quantification of hippocampal volume and signal significantly increased the detection of signs of HS.

In our series, only 1 patient with visual signs of HS had normal volumetry and signal quantification. In this patient, by visual analysis, we observed normal hippocampal volume and a localized hyperintense signal in the body of the hippocampus, contralateral to the epileptic focus determined by EEG. This pattern of subtle and localized hippocampal abnormality (restricted to the hippocampal body) is not commonly seen. We believe that this discordance could be overcome if more sections of the coronal MR imaging sequence were added to the relaxometry analysis. However, this addition would add time to the procedure and would probably result in limited improvement in the detection rate $(<1 \%)$.

The increase of patients with detectable MR imaging abnormalities compatible with the site of the seizure-onset origin is significantly important for drug-resistant focal epilepsies. With quantitative analysis of 1.5T MRI, a group of patients with MTLE remains with no detectable structural abnormalities even when HS is confirmed after surgical removal of the mesial temporal structures. ${ }^{22}$ For patients with drug-resistant MTLE and normal MR imaging findings, invasive procedures, which have high cost and morbidity, are often necessary to evaluate the potential target for surgical intervention, ${ }^{23}$ and yet for these individuals, the rate of seizure freedom is lower than that for those with MRI signs of HS. ${ }^{24,25}$ Histopathology of HS is only found in a limited number of patients with MTLE with normal MR imaging findings undergoing surgery, but those with positive hippocampal pathology are the ones with better surgical outcomes. ${ }^{24,26}$ It is necessary to improve noninvasive techniques that could more efficiently select the individuals with MTLE with subtle HS to better select surgical patients and improve outcomes. In this context, we believe that the use of hippocampal quantification measures in 3T MRI, as we showed in this article, can help in the recognition of these individuals with hippocampal sclerosis not detected by visual analysis who could benefit from surgical procedures for control of drugresistant seizures. The complete benefit of the use of hippocampal quantification measurements in 3T MRI in the presurgical evaluation of patients with drug-resistant MTLE could be assessed with follow-up and surgical outcome data of these patients.

In this study, we were not able to satisfactorily determine the specificity of the quantification procedures in the detection of HS. To achieve this goal, a correlation of these findings with the criterion standard, histopathology, would be necessary. We were only able to evaluate the histopathologic findings in 10\% (19 patients) of our group. In the sample of our patients undergoing surgery, the specificity of hippocampal volumetry and T2 signal quantification was $100 \%$ (all patients with abnormal hippocampal volume or T2 signal had confirmed histopathology of HS, and the only patient with a normal hippocampus by these quantification techniques had normal histopathology). However, this specificity is biased because all patients who underwent surgery in our group had similar findings in both visual and quantification analysis, and the visual analysis was used in the selection of the patients for the surgical procedure. However, a good correlation of volume and signal abnormalities detected by quantification analysis and histopathology of HS has been previously demonstrated. ${ }^{27}$ Also, in our group of patients, the laterality of abnormal hippocampal volume and signal was highly concordant with the laterality of the epileptic focus defined by EEG recordings.

Currently, most studies of hippocampal volumetry apply manual hippocampal delimitation, and whether manual ${ }^{28}$ or automatic analysis has higher sensitivity and specificity is still debatable. $^{12,13}$ Despite this controversy, in the clinical context, the quantification of hippocampal abnormalities must be as fast and practical as possible. In this article, we used automated volumetry and a simple manual signal quantification in which it is necessary only to define a small region of interest in 3 sections of the T2 scan of each patient, without the need to define precisely the borders of the hippocampus. We think that this optimized hippocampal quantification protocol can be easily applied to all patients with drug-resistant focal epilepsies as a parallel and additional analysis for the routine evaluation of MRI in specialized epilepsy centers. Although FreeSurfer software is not approved for clinical use by the US Food and Drug Administration, as far as we know, there is at least 1 software approved for automated volumetric analyses of brain MRI. ${ }^{27}$ However, this has only been evaluated for the detection of hippocampal abnormalities on $1.5 \mathrm{~T}$ scanners. ${ }^{13}$

In 1.5T MRI, a good correlation of volume and signal abnormalities detected by quantification analysis and histopathology of HS has been demonstrated. ${ }^{29}$ More recently, histologic studies have proposed that different patterns of neuronal loss in the hippocampus might be associated with different seizure outcome after surgical removal of the temporal lobe. ${ }^{30}$ Also, it has been shown that quantitative measures in 3T MRI can demonstrate ultrastructural details of HS pathology not detectable with lower field scanners. ${ }^{17,31}$ These in vivo quantitative measurements of this detailed HS pathology on 3T MR imaging scanners might contribute, in the future, to the better selection of patients with drug-resistant MTLE who will benefit from surgical procedures. 


\section{CONCLUSIONS}

In MRI performed with a 3T scanner and visually inspected by imaging experts, quantification of hippocampal volume and T2 signal can increase the detection of signs of HS in approximately $28 \%$ of patients with MTLE. Today, these MR imaging quantification methods are easily available and not very time-consuming, and they could be used as routine diagnostic tools for patients with drug-resistant focal epilepsies and visually normal MRI findings after further validation for clinical use.

Disclosures: Ana C. Coan—RELATED: Grant: Fundação de Amparo à Pesquisa do Estado de São Paulo (FAPESP) (São Paulo Research Foundation), Comments: PhD scholarship, UNRELATED: Payment for Lectures (including service on Speakers Bureaus): Novartis. Bruno Kubota—RELATED: Grant: FAPESP, Comments: scientific scholarship. Felipe P.G. Bergo-RELATED: Grant: FAPESP, Comments: Postdoctoral scholarship funding from a government agency. Postdoctoral project includes the subject of this study but is not limited to it, UNRELATED: Grants/Grants Pending: FAPESP. Fernando Cendes-RELATED: Grants: FAPESP and Conselho Nacional de Desenvolvimento Científico e Tecnológico, Brazil, ${ }^{*}$ Comments: FAPESP grants 2005/ 56578-four and 2009/54552-9. *Money paid to the institution.

\section{REFERENCES}

1. Engel J. Introduction to temporal lobe epilepsy. Epilepsy Res 1996;26:141-50

2. Van Paesschen W, Connelly A, King MD, et al. The spectrum of hippocampal sclerosis: a quantitative magnetic resonance imaging study. Ann Neurol 1997;41:41-51

3. Cendes F, Leproux F, Melanson D, et al. MRI of amygdala and hippocampus in temporal lobe epilepsy. J Comput Assist Tomogr 1993;17:206-10

4. Van Paesschen W, Sisodiya S, Connelly A, et al. Quantitative hippocampal MRI and intractable temporal lobe epilepsy. Neurology 1995;45:2233-40

5. Sloviter RS. The neurobiology of temporal lobe epilepsy: too much information, not enough knowledge. C R Biol 2005;328:143-53

6. Jackson GD, Berkovic SF, Tress BM, et al. Hippocampal sclerosis can be reliably detected by magnetic resonance imaging. Neurology 1990;40:1869-75

7. Berkovic SF, Andermann F, Olivier A, et al. Hippocampal sclerosis in temporal lobe epilepsy demonstrated by magnetic resonance imaging. Ann Neurol 1991;29:175-82

8. Cendes F, Andermann F, Gloor P, et al. MRI volumetric measurements of amygdala and hippocampus in temporal lobe epilepsy. Neurology 1993;43:719-25

9. Jackson GD, Connelly A, Duncan JS, et al. Detection of hippocampal pathology in intractable partial epilepsy. Increased sensitivity with quantitative magnetic resonance T2 relaxometry. Neurology 1993;43:1793-1793-99

10. Bernasconi A, Bernasconi N, Caramanos Z, et al. T2 relaxometry can lateralize mesial temporal lobe epilepsy in patients with normal MRI. Neuroimage 2000;12:739-46

11. Duncan JS. Neuroimaging methods to evaluate the etiology and consequences of epilepsy. Epilepsy Res 2002;50:131-40

12. Hammers A, Heckemann R, Koepp MJ, et al. Automatic detection and quantification of hippocampal atrophy on MRI in tempo- ral lobe epilepsy: a proof-of-principle study. Neuroimage 2007;36:38-47

13. Farid N, Girard HM, Kemmotsu N, et al. Temporal lobe epilepsy: quantitative MR volumetry in detection of hippocampal atrophy. Radiology 2012;264:542-50

14. Knake S, Triantafyllou C, Wald LL, et al. 3T phased array MRI improves the presurgical evaluation in focal epilepsies: a prospective study. Neurology 2005;65:1026-31

15. Briellmann, RS, Syngeniotis, A, Jackson, GD. Comparison of hippocampal volumetry at $1.5 \mathrm{~T}$ and at $3 \mathrm{~T}$. Epilepsia 2001;42:1021-24

16. Scorzin JE, Kaaden S, Quesada CM, et al. Volume determination of amygdala and hippocampus at 1.5 and 3.0 T MRI in temporal lobe epilepsy. Epilepsy Res 2008;82:29-37

17. Howe KL, Dimitri D, Heyn C, et al. Histologically confirmed hippocampal structural features revealed by $3 T$ MR imaging: potential to increase diagnostic specificity of mesial temporal sclerosis. AJNR Am J Neuroradiol 2010;31:1682-89

18. Proposal for revised classification of epilepsies and epileptic syndromes: Commission on Classification and Terminology of the International League Against Epilepsy. Epilepsia 1989;30:389-99

19. McLachlan RS, Nicholson RL, Black S, et al. Nuclear magnetic resonance imaging, a new approach to the investigation of refractory temporal lobe epilepsy. Epilepsia 1985;26:555-62

20. Von Oertzen J, Urbach H, Jungbluth S, et al. Standard magnetic resonance imaging is inadequate for patients with refractory focal epilepsy. J Neurol Neurosurg Psychiatry 2002;73:643-47

21. Berkovic SF, McIntosh AM, Kalnins RM, et al. Preoperative MRI predicts outcome of temporal lobectomy: an actuarial analysis. Neurology 1995;45:1358-63

22. Jackson GD, Kuzniecky RL, Cascino GD. Hippocampal sclerosis without detectable hippocampal atrophy. Neurology 1994;44:42-46

23. Cohen-Gadol AA, Bradley CC, Williamson A, et al. Normal magnetic resonance imaging and medial temporal lobe epilepsy: the clinical syndrome of paradoxical temporal lobe epilepsy. J Neurosurg 2005;102:902-09

24. Bell ML, Rao S, So EL, et al. Epilepsy surgery outcomes in temporal lobe epilepsy with a normal MRI. Epilepsia 2009;50:2053-60

25. Schwartz TH, Jeha L, Tanner A, et al. Late seizures in patients initially seizure free after epilepsy surgery. Epilepsia 2006;47:567-73

26. Sylaja PN, Radhakrishnan K, Kesavadas C, et al. Seizure outcome after anterior temporal lobectomy and its predictors in patients with apparent temporal lobe epilepsy and normal MRI. Epilepsia 2004;45:803-08

27. Brewer JB. Fully-automated volumetric MRI with normative ranges: translation to clinical practice. Behav Neurol 2009;21:21-28

28. Pardoe HR, Pell GS, Abbott DF, et al. Hippocampal volume assessment in temporal lobe epilepsy: how good is automated segmentation? Epilepsia 2009;50:2586-92

29. Cascino GD, Jack CR, Parisi JE, et al. Magnetic resonance imagingbased volume studies in temporal lobe epilepsy: pathological correlations. Ann Neurol 1991;30:31-36

30. Blümcke I, Pauli E, Clusmann H, et al. A new clinico-pathological classification system for mesial temporal sclerosis. Acta Neuropathol 2007;113:235-44

31. Hanamiya M, Korogi Y, Kakeda S, et al. Partial loss of hippocampal striation in medial temporal lobe epilepsy: pilot evaluation with high-spatial-resolution T2-weighted MR imaging at 3.0 T1. Radiology 2009;251:873-81 